\title{
Falar ou calar: eis a questão para enfermeiros
}

\author{
Speak or shut up: that is the question for nurses
}

Hablar o callar: esa es la cuestión para los enfermeros

\section{Resumo}

Introdução: A forma como as relações interprofissionais em saúde se estabelece, podem gerar erros e resultar em danos aos pacientes. Objetivos: examinar a capacidade dos enfermeiros para manifestarem-se frente às falhas de assistência ao paciente com potencial para eventos adversos; e, identificar os facilitadores capazes de potencializar a voz dos enfermeiros, frente aos atos inseguros em curso, praticados por profissionais da saúde em dois hospitais da região metropolitana de Porto Alegre/RS. Metodologia: realizado um estudo de caso múltiplo e incorporado. A coleta de dados entre julho a agosto de 2020, por meio de entrevista semiestruturada, virtual e gravada; para análise dos dados adotou-se o método de análise de Laurence Bardin. Resultados: a capacidade de enfermeiros manifestarem suas preocupações relacionadas à segurança do paciente dependeu de alguns fatores além da gravidade do dano, tais quais, o aspecto pessoal, a pessoa a ser contestada ou questionada, o profissional (onde, abordar a equipe médica foi mais difícil que a técnica de enfermagem), e a situação ou contexto. Os potenciadores da fala também emergiram, principalmente: pessoal, experiência, conhecimento, liderança, gestão e processos. Conclusão: características pessoais, tal qual a maturidade, e conhecimento, auxiliaram na tomada de decisão, enquanto a interdisciplinaridade apareceu discretamente nos discursos, reforçando a necessidade do trabalho em equipe com este foco, a fim do compartilhamento de conhecimento e apoio nas incertezas.

Palavras-chave: Comunicação; Barreiras de comunicação; Segurança do paciente; Erros médicos.

\begin{abstract}
Introduction: The way how inter-professional relationships in health are established can generate errors and harm to patients. Objective: examine the nurses' ability to manifest themselves in the face of failures in patient care with the potential for adverse events; and, identify facilitators capable of enhancing the voice of nurses, in the face of ongoing unsafe acts, practiced by health professionals in two hospitals in the metropolitan region of Porto Alegre / RS. Methodology: a multiple and embedded case study was carried out. Data collection between July and August 2020, through semi-structured, virtual, and recorded interviews; for data analysis, the method of analysis of Laurence Bardin was adopted. Results: the ability of nurses to express their concerns related to patient safety depended on some factors in addition to the severity of the damage, such as the personal aspect, the person to be challenged or questioned, the professional (where, approaching the medical team was more difficult than the nursing technician), and the situation or context. Speech enhancers also emerged, mainly: personnel, experience, knowledge, leadership, management, and processes. Conclusion: personal characteristics, such as maturity, and knowledge, helped in decision making, while interdisciplinarity appeared discreetly in the speeches, reinforcing the need for teamwork with this focus, in order to share knowledge and support in uncertainties.
\end{abstract}

Keywords: Communication; Communication barriers; Patient safety; Medical errors. 


\begin{abstract}
Resumen
Introducción: La forma en que se establecen las relaciones interprofesionales en salud, pueden generar errores y resultar daños para los pacientes. Objetivo: examinar la capacidad de los enfermeros para manifestaren se ante fallas en la atención al paciente con potencial de eventos adversos; e, identificar facilitadores capaces de realzar la voz de los enfermeros, ante los actos inseguros en curso, practicados por profesionales de la salud en dos hospitales de la región metropolitana de Porto Alegre / RS. Metodología: se realizó un estudio de caso múltiple e integrado. Recolección de datos entre julio y agosto de 2020, a través de entrevistas semi-estructuradas, virtuales y grabadas; para el análisis de datos, se adoptó el método de análisis de Laurence Bardin. Resultados: la capacidad de los enfermeros para expresar sus inquietudes relacionadas con la seguridad del paciente dependió de algunos factores además de la gravedad del daño, como el aspecto personal, la persona a ser contestada o cuestionada, el profesional (donde, acercarse al equipo médico fue más difícil que el técnico de enfermería), y la situación o contexto. También surgieron potenciadores del habla, principalmente: personal, experiencia, conocimiento, liderazgo, gestión y procesos. Conclusión: las características personales, como la madurez y el conocimiento, ayudaron en la toma de decisiones, mientras que la interdisciplinariedad apareció discretamente en los discursos, reforzando la necesidad del trabajo en equipo con este enfoque, para compartir conocimientos y apoyos en las incertidumbres.
\end{abstract}

Palabras clave: Comunicación; Barreras de comunicación; Seguridad del paciente; Errores médicos.

\title{
1. Introdução
}

Agravos e danos aos pacientes relacionados a problemas durante a prestação de serviços em saúde têm demonstrado elevada frequência e gravidade desencadeando a mobilização tanto de órgãos governamentais, quanto não governamentais. No Brasil, foi publicada a Portaria n ${ }^{\circ} 529$, de 01 de abril de 2013, que institui o Programa Nacional de Segurança do Paciente (PNSP), e definiu evento adverso (EA) como o incidente que causa dano ao paciente, ou seja, resulta em comprometimento de estrutura ou função do corpo podendo ser físico, social ou psicológico (Brasil Ministério da saúde, 2013).

Trabalhos têm mostrado a ocorrência de eventos adversos em hospitais (Agency for Healthcare Research and Quality, 2017; Figueiredo et al., 2018). No âmbito da saúde, o fator humano tem contribuído de maneira significativa com os erros, por isso, defende-se a importância da abordagem dos problemas referentes a atitudes e comportamentos que ameaçam o desempenho do cuidado da equipe de saúde, como as questões que envolvem comunicação e comportamentos destrutivos no ambiente de trabalho (TartagliaReis et al., 2018).

Portanto os relacionamentos entre os profissionais da saúde têm sido determinante para a segurança do paciente, ao passo que as barreiras fortemente inseridas nestas relações, são capazes de se sobressair ao cuidado seguro, um exemplo disso, é a hierarquia (Olde Bekkink et al., 2018).

Ainda sobre relacionamento interprofissional, uma síntese narrativa explorou as barreiras modificáveis e intervenções educacionais sobre a capacidade de falar e desafiar a autoridade em ambiente cirúrgico. Os dados foram agrupados em 3 categorias distintas conforme o desenho do estudo e incluídas publicações entre 2003-2017, nestes, um dos temas mais estudados foi a hierarquia, principalmente na abordagem qualitativa (Pattni et al., 2019).

Corroborando com a temática, uma meta síntese interpretativa entre 2005 e 2015, investigou o comportamento de voz seguro e sua influência na prática clínica na visão de diferentes profissionais da saúde, na qual alguns temas foram identificados, entre eles: hierarquia e dinâmicas de poder; comunicação aberta não efetiva; e expectativas imbuídas. Todos relataram medos atribuídos a questões sociais e hierárquicas, que cercam os relacionamentos. Em particular, os enfermeiros relatam muitos medos relacionados aos comportamento de voz seguro, que podem ser exacerbados pelas expectativas sociais dos enfermeiros, resultando na hesitação generalizada em se manifestar e, portanto, a eficácia mínima da comunicação aberta (Morrow et al., 2016).

Como parte da equipe, destaca-se a força de trabalho do enfermeiro que permanece durante maior período frente às ações de cuidado assistencial, cujo papel histórico e contemporâneo na promoção da segurança do paciente, entre 1900 e 2015 , 
foi aumentando concomitante à complexidade do atendimento. Neste contexto, os enfermeiros desenvolveram uma identidade profissional, focando, muitas vezes, nas preocupações e soluções de segurança (Kowalski \& Anthony, 2017).

Frente a isso, falar sobre as preocupações quando se acredita que uma situação é arriscada ou, um funcionário inicia um curso de ação inseguro, demonstra ser uma das dificuldades encontradas por profissionais que prestam assistência ao paciente. Entretanto, a criação de um ambiente seguro não é uma tarefa fácil, dependente de muitos fatores e pessoas que sustentarão a prestação de serviço em saúde de qualidade. Considerando o conhecimento e envolvimento do enfermeiro com a segurança do paciente, o objetivo desta pesquisa foi: examinar a capacidade destes profissionais para manifestarem-se frente às falhas de assistência ao paciente com potencial para eventos adversos; e, identificar os facilitadores capazes de potencializar a própria voz, frente aos atos inseguros em curso, praticados por outros profissionais da saúde.

\section{Metodologia}

Realizou-se um estudo de caso múltiplo incorporado (Yin, R.K., 2015), de abordagem qualitativa, a coleta de dados ocorreu no período de julho de 2020 a agosto de 2020 em dois Hospitais de Porto Alegre/RS, um público e outro privado, sendo apenas o último com certificação internacional de qualidade em saúde e/ou segurança do paciente desde 2002.

Participaram da pesquisa 23 enfermeiros assistenciais da emergência e unidades de internação, atendendo ao seguinte critério de inclusão: atuar na assistência direta ao paciente há pelo menos dois anos na instituição, tempo mínimo e razoável para um funcionário participar de treinamentos obrigatórios, adaptação a rotinas, normas e cultura organizacional; referente aos critérios de exclusão: enfermeiros com históricos de afastamento nos últimos dois meses prévios ao período da coleta de dados, por acreditar que isso podia contribuir com o viés de memória, já que as informações foram baseadas em histórias de eventos retrospectivos. A replicação abrangeu duas unidades de análise (hospital privado acreditado e Hospital público sem acreditação), composta por seis enfermeiros de cada departamento (recrutados de forma aleatória e por conveniência na emergência e unidade de internação).

Para início da coleta de dados, garantiu-se as aprovações dos Comitês de Ética em Pesquisa da Universidade Luterana do Brasil (ULBRA), Hospitais coparticipantes, e mediante assinatura do Termo de Consentimento Livre e Esclarecido (TCLE). Utilizou-se entrevista semiestruturada, virtual e gravada, com enfermeiros das distintas unidades e organizações. O instrumento de coleta de dados foi composto por duas partes: a primeira com 8 perguntas fechadas com o objetivo de levantamento do perfil dos participantes e a segunda tratou-se de 7 questões abertas. Para formulação do instrumento foi consultado o questionário da Agency for Healthcare Research and Quality (AHRQ), destinado a avaliar a cultura de segurança de hospitais, por ser validado e implementado por centenas de hospitais nos Estados Unidos e internacionalmente, desde 2004 (AHRQ. Agency for Healthcare Research and Quality, 2004), e também um roteiro de entrevista utilizado em artigo que investigou as barreiras e facilitadores da comunicação interprofissional (Olde Bekkink et al., 2018).

Para análise dos dados foi adotado o método de análise de Laurence Bardin (2016). A codificação das unidades de análise e subunidades para a tabulação dos dados foi a seguinte: (H@- hospital privado certificado; H- hospital público sem certificação; E- emergência; UI- unidade de internação), quanto aos participantes da pesquisa o código utilizado foi P, mais a numeração de 1 a 24 correspondente à ordem em que as entrevistas ocorreram. Os facilitadores capazes de potencializar a manifestação dos enfermeiros frente às preocupações e dúvidas sobre a assistência segura, surgiram dos relatos sobre a própria atitude de falar ou não.

Para análise de dados e reflexão dos achados realizou-se o aprofundamento e comparação embasados nas mais recentes evidências científicas sobre segurança do paciente, eventos adversos e comunicação, a nível nacional e internacional. 


\section{Resultados e Discussão}

O perfil dos participantes da pesquisa se caracterizou por profissionais experientes: 17 com 10 anos ou mais na função de enfermeiro, 4, entre 5 a 9 anos, e apenas 2 deles, 4 e 3 anos; entretanto, estes dois menos experientes, já possuíam vínculo empregatício com a instituição, respectivamente, há 9 e 25 anos. Portanto, todos possuíam vivência hospitalar e a ambientação à cultura organizacional. A faixa etária predominante foi entre 30 e 40 anos (n=14); na sequência, acima de 40 anos (n=7); e 2 enfermeiros, 28 e 29 anos.

Quanto aos demais aspectos avaliados, 17 participantes eram do diurno e 6 do noturno; 19, sexo feminino e 4 masculinos; 22 com pós-graduação (17 em nível de especialização, 4, mestrado, e 1 doutorado) e um, somente graduação.

Perfis de idade e tempo de experiência demonstraram relevância em outro estudo, com alunos de pós-graduação em enfermagem, na China, quando atitudes negativas em relação à cultura de segurança variaram de acordo com a idade e experiência (Kong et al., 2019).

Entre as atitudes que comprometem a segurança do paciente, consta a tomada de decisão do enfermeiro de falar e interromper um ato inseguro em curso, praticado por outro profissional. Frente ao risco iminente de morte ou dano grave ao paciente, quando não existe dúvida de que isso possa acontecer, todos participantes responderam que se manifestam.

Porém, de acordo com o Gráfico 1, a tomada de decisão entre contestar ou desafiar outros profissionais frente à situação de menor risco à segurança do paciente, para 10 enfermeiros, foi colocada na balança, e pesaram fatores, aquém ou além da gravidade do dano.

Gráfico 1. Falar ou calar diante do risco de menor gravidade ao paciente.

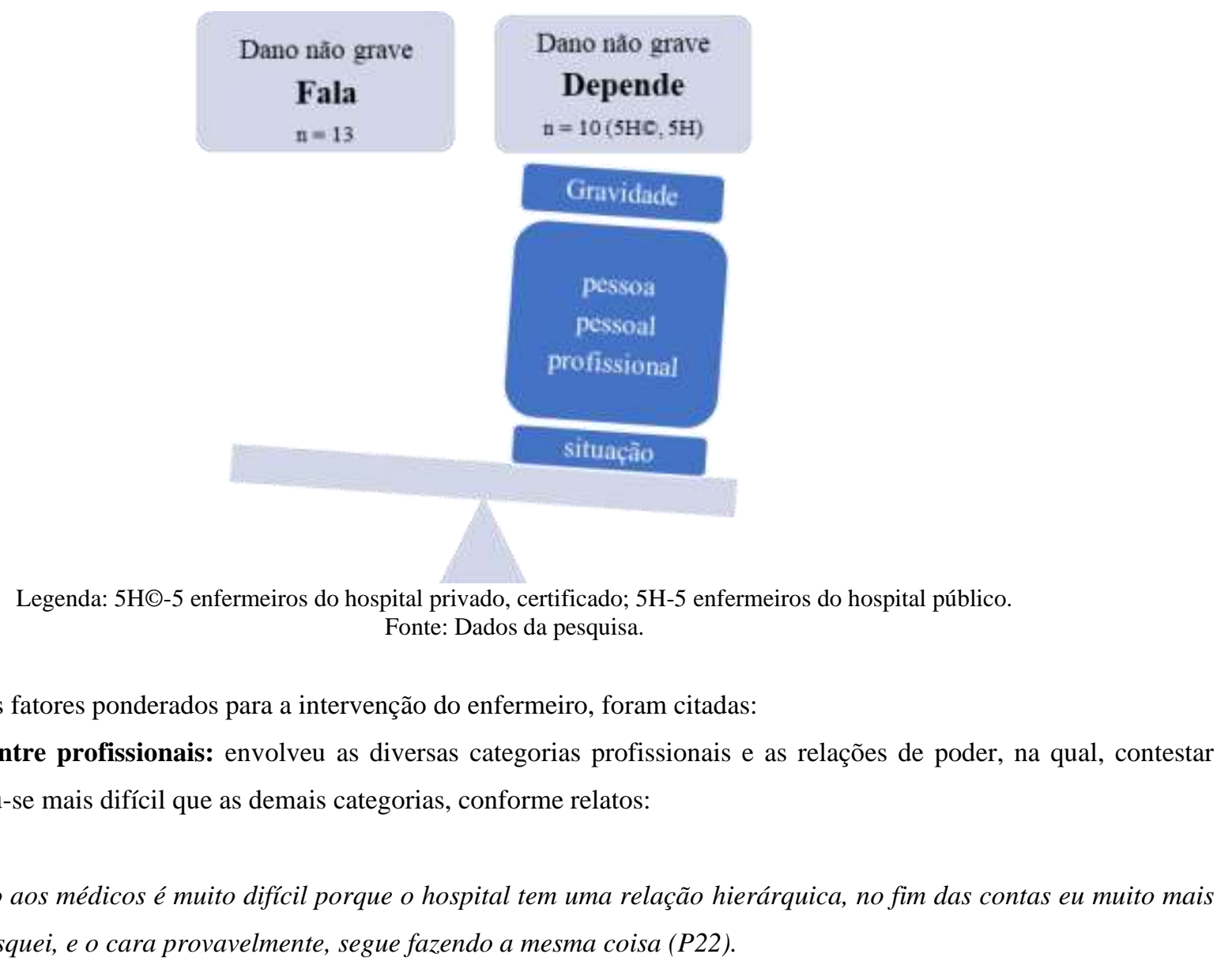

me arrisquei, e o cara provavelmente, segue fazendo a mesma coisa (P22). 
Não me sinto com o mesmo tipo de liberdade para chegar e contestar o médico, do que o técnico (P24).

As questões pessoais: revelou-se pela insegurança, cansaço, impotência, dúvidas, incertezas, medo e timidez, conforme relato de P14: "primeiro conhecer, entender como é o funcionamento, como acontece, quem são as pessoas, provavelmente, eu sou uma pessoa tímida, eu não consigo, eu não tenho esse perfil extremamente comunicador, então eu preciso sentir o ambiente para também me sentir segura de tomar uma certa atitude junto a equipe, mesmo tendo conhecimento”.

A pessoa a ser contestada: um dos determinantes para contestar outro profissional, foi justamente, quem seria este, exemplificado na fala de P8: "o certo seria com todo mundo, mas aí vou ser sincera que depende do médico, se é um médico que dá abertura, que é mais tranquilo, tu fala com jeitinho, que tu sabe que não é tão fácil”.

A situação, ou contexto: ponderar a situação de maneira geral, pensar e avaliar o contexto antes de agir, preocupar-se em não expor um profissional na frente do paciente, somou-se aos demais motivos dos enfermeiros manterem-se calados frente a situações de risco ao paciente praticado por outro profissional:

Num primeiro momento eu paro e penso, eu peso a situação (P16).

Dependendo da situação, eu não falo na frente do paciente, pois ele pode pensar: "essas mulheres não sabem nem o que estão fazendo" (P21).

Chega uma hora que eu me sinto cansada de falar para todo mundo. Eu me sinto impotente em relação àquelas situações (referente à violação de regras de precaução para pacientes em isolamento) (P24).

À luz de nossos achados (Kiesewetter et al., 2018), revelaram intenções comportamentais de estudantes de medicina após terem observado ou causado um erro hipotético. Estas, foram definidas de acordo com: quem cometeu o erro, a presença ou ausência de testemunhas, e o resultado do erro para o paciente. Um outro estudo identificou alguns condicionantes às dúvidas sobre expressar opiniões, encontrando a incerteza, não só sobre a pessoa, mas ao conflito à definição adequada de situações particulares a serem julgadas (Martin et al., 2018). Sobre a influência da interpretação de profissionais sobre comportamentos de colegas no atendimento aos pacientes, Lee e Doran (2017) também corroboram com esta pesquisa.

Diversos estudos investigam motivos e percepções de profissionais da saúde para a notificação e ou relato de incidentes, cujos resultados circundam: a preocupação com os relacionamentos de trabalho (Hammoudi et al., 2018; Levine et al., 2020; Varallo et al., 2018); a imprevisibilidade de reação da pessoa a ser questionada e a falta de clareza sobre riscos da situação (Schwappach et al., 2019). Desta forma, falar sobre a segurança do paciente, assim como em nossa pesquisa, nem sempre se caracteriza por um mecanismo simples e instintivo, sendo influenciada diretamente pelos relacionamentos interpessoais.

\section{Os potencializadores da fala dos enfermeiros}

Facilitadores potencializaram a fala do enfermeiro frente às preocupações com a segurança do paciente, estes, estiveram associados à capacidade de manifestação destes profissionais diante de falhas assistenciais ou atos inseguros praticadas por colegas de trabalho.

Ao que pesem os fatores condicionantes à tomada de decisão, no qual, para determinados participantes dependeu do “GPS” (gravidade, pessoa, profissional, pessoal e situação), as principais categorias que impulsionaram os enfermeiros à fala, em ordem decrescente da frequência de citação, foram: pessoal, conhecimento, experiência, liderança, gestão e processos, todas elas consideradas atributos fundamentais à decisão de falar, estes dados podem ser visualizados no gráfico 2. 
Gráfico 2. Facilitadores da fala dos enfermeiros frente a situações de risco à segurança do paciente.

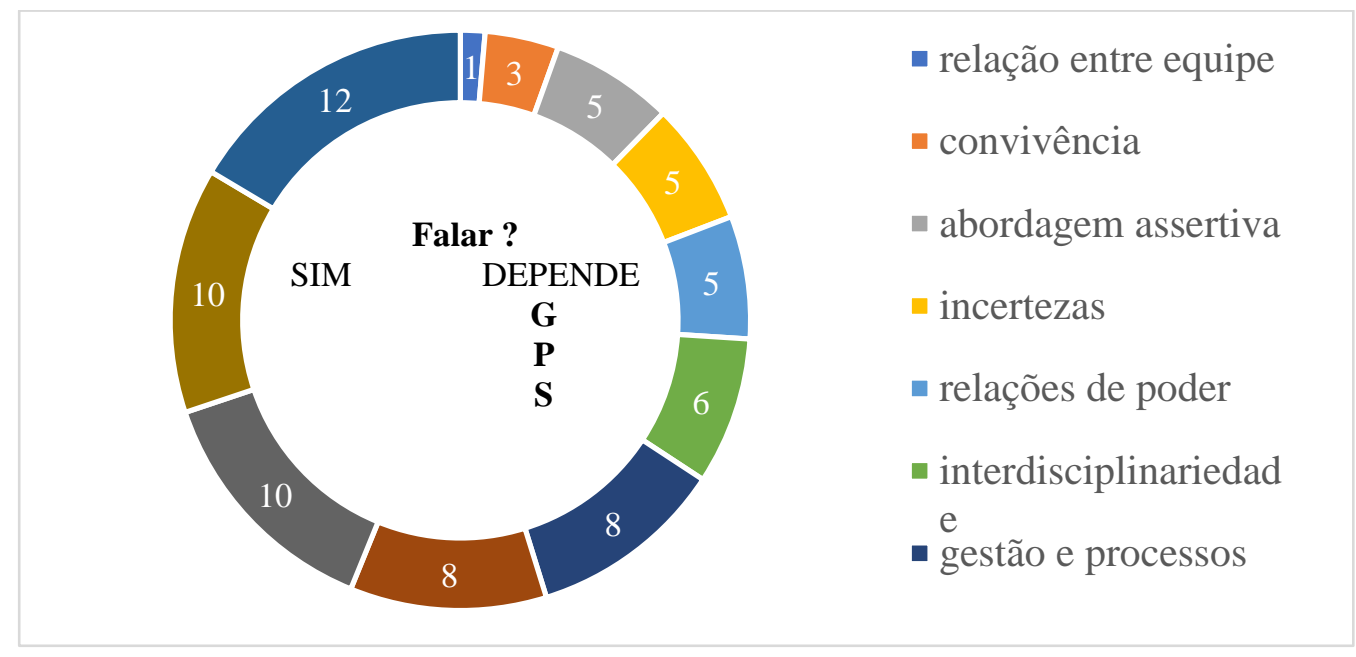

Legenda: G gravidade; P pessoa, pessoal, profissional; S situação.

Fonte: Dados da pesquisa.

Dos facilitadores, cinco categorias foram as mais significativas: Pessoal, conhecimento, experiência, liderança, gestão e processos.

Pessoal: características pessoais auxiliaram no estabelecimento das relações:

Acho que é um pouco meu de comunicar e conseguir conversar (P5).

Eu sinto que todos me respeitam do jeito que eu sou. Então, eu trouxe para a minha vida que não é como tu chega nas pessoas, todo mundo tem que aceitar do jeito que tu é e tu tem que aceitar do jeito que as pessoas são (P15).

O médico que vai entrar de plantão é mais tranquilo, então espera o outro ir embora (P22).

Um dos atributos pessoais mais citados foi a maturidade, estando associada ao desenvolvimento de habilidades para resolução de problemas sem haver confrontos, e de conversar abertamente com a equipe:

Maturidade para solucionar de uma maneira não de confronto (P10).

Em qualquer nível hierárquico, e com profissionais diferentes, mas eu acho que é uma questão de maturidade (P11).

Conhecimento: esse facilitador proporcionou segurança aos enfermeiros:

Antigamente eu não fazia, mas hoje eu me empoderei, por causa do conhecimento (P1).

Só vou questionar algo se eu tiver algum embasamento, ou se eu tiver dúvida por falta de conhecimento (P11).

Quando tu vai argumentar com os médicos tu tem que ter o mínimo de conhecimento do no qual tu vai abordá-lo (P18).

Experiência: esta categoria foi adquirida conforme o tempo de vivência na prática profissional:

Com o tempo que eu tenho de experiência, é mais fácil para mim, porque com a carga que eu tenho de experiência, de falar que eu, não vou fazer(P2). 
Com o passar dos anos, com mais experiência, mais maturidade, eu me sinto à vontade (P9).

É uma prática, hoje é tranquilo, é que hoje, 8 anos (P13).

Liderança: o enfermeiro como referência da equipe, marcou o discurso de alguns participantes da pesquisa:

Residentes de medicina, fonoaudióloga, terapia ocupacional, assistente social e psicologia tinham como referência a enfermeira da unidade (P10).

Minha função ali dentro é ser um advogado para o paciente, proteger o paciente e gerenciar o cuidado (P19).

Gestão e processos: gestão organizacional incluindo vínculo empregatício, protocolos e rotinas e cultura de segurança determinou comportamento, promoveu autonomia e empoderou profissionais:

Com protocolo é bem mais fácil (P3).

A instituição dá um respaldo... não é uma coisa punitiva (P5).

Se o cara chega de nariz empinado, o meio derruba ele (P13).

A gente tem muita autonomia... todos são contratados, inclusive médicos (P14).

Interdisciplinaridade: apoio interdisciplinar auxiliou frente ao desconhecido, à dúvida, insegurança e falta de aceitação da opinião do enfermeiro:

O outro não aceita a minha posição, a gente tem muito de envolver o plantão, envolve a nossa supervisora (P6).

Vou tentando, não deu com um eu vou com outro, chamo plantão que estiver no time de resposta rápida. Vou pedindo para um e para outro até que o outro responde (P8).

Apesar da frequência menor de citação, as demais categorias (Incertezas, abordagem assertiva, relações de poder, convivência e relação entre equipe), fizeram diferença na tomada de decisão dos enfermeiros.

Incertezas: dúvida e aquilo que não é habitual, impulsionou o questionamento:

O que eu não sei, eu pergunto (P1).

Não faço com dúvida (P4).

Alguma coisa que não estão acostumados (referente aos membros da equipe de saúde), não usual, eles vão ir atrás e vão questionar (P12).

Abordagem assertiva: adaptação do discurso levando em consideração o contexto e a pessoa, sem deixar de intervir em prol do paciente, mostrou-se fundamental para evitar conflitos, resolver problemas e preservar relacionamentos:

Eu aprendi a falar sem magoar as pessoas (P8).

Tem que avaliar o colega, avaliar a situação para também não ser errôneo nesta abordagem (P18).

Vou comendo pelas beiradas... daí eu falei: doutor ela está um pouquinho hipotensa, seria interessante um volume (P21). 
Tem situações que a gente tem um cuidado maior com as palavras... como vai se direcionar (P23).

Relações de poder: a hierarquia dita necessária, expressa nas inferências, e a responsabilidade de gestão da equipe, justificou a categoria como um facilitador:

Em relação aos técnicos de enfermagem eu acredito que seja mais fácil porque como a gente é responsável por eles (P12).

A hierarquia é necessária, eu acho que existe um pouco de desrespeito, que tem profissionais que não observam essa hierarquia, e se sentem protegidos em uma instituição (P24).

Convivência: o tempo de vivência com a mesma equipe, facilitou a comunicação entre os profissionais, conforme a fala de P14: “a questão de ser uma equipe que trabalha junto há muito tempo, isso também facilita, então tu já conhece vários médicos, teus colegas".

Relação entre equipe: destacou-se o trabalho cooperativo, papéis bem definidos dos profissionais, as preocupações com a própria reputação e o paciente, segundo relato de P14: “a equipe trabalha de uma forma bem cooperativa, todos sabem seus papéis, e mediante uma situação de risco ao paciente a gente consegue ter autonomia... se tem essa abertura para a gente discutir".

A decisão entre falar e calar sobre situações de risco à segurança do paciente mostrou-se, apesar das barreiras, possível e passível de ser modificada pela influência dos facilitadores. Para tanto, os profissionais cujos enfermeiros discutiram essas questões variou entre técnicos de enfermagem e médicos. No $\mathrm{H} \odot$, enfermeiros citaram menos os médicos (6 vezes), enquanto no $\mathrm{H}$, foram citados 10 vezes.

Referente às subunidades, o comportamento relacionado à pessoa a ser contestada, se modificou por uma maior frequência de técnicos de enfermagem abordados pelo enfermeiro nas unidades de internação. Em contrapartida, na E, as necessidades de abordagens se referiram aos médicos. É possível que esta ocorrência se dê pela formatação das equipes nas subunidades, no qual, a E tem em sua composição, a presença de médico 24 horas, já a UI, caracteriza-se por visitas médicas de rotina ou atendimentos quando solicitado.

Apesar da existência de barreiras à comunicação entre equipe, isso não foi suficiente para silenciar os enfermeiros, pois, muitos facilitadores surgiram, especificamente no $\mathrm{H}$, tais quais a autonomia fornecida pela instituição hospitalar, assim descrito por P12: “É por causa da instituição, toda equipe de enfermagem lá é muito autônoma... a gente tem muito controle de todas as salas... então os pacientes não são dos médicos”.

Em reforço à voz dos enfermeiros, a interdisciplinaridade, citada cinco vezes no H@ e no H 1, apareceu no compartilhamento da tomada de decisão com outros profissionais, de acordo com o relato de P4: "Quando não tenho o conhecimento... eu busco na farmacêutica, que é uma parceira pra isso”.

Poucas pesquisas investigam facilitadores à capacidade de se manifestar de profissionais da saúde, diante de situações arriscadas ao paciente (Campbell et al., 2019; Martin et al., 2018). De uma maneira mais ampla, os facilitadores evidenciados na literatura costumam referir-se à comunicação interprofissional (Cunningham et al., 2018; Grade et al., 2019; Manias, 2018; Olde Bekkink et al., 2018; Rönnerhag et al., 2019).

Aproximando-se de nossos achados, no que tange os facilitadores: interdisciplinaridade, ação comunicativa, conhecimento, gestão e processos, abordagem assertiva e liderança, a literatura contempla alguns facilitadores à comunicação 
eficaz, tais quais as oportunidades de comunicação e transferência de conhecimento, habilidades e sistemas de comunicação (Cunningham et al., 2018). Outros contributivos relacionados aos ambientes seguros, segundo o estudo de Rönnerhag et al. (2019), estão a promoção do trabalho em equipe interprofissional, construção de capacidades envolvendo a percepção de profissionais de saúde, e a elucidação de estratégias relevantes.

No que concerne às categorias convivência e pessoal, a confiança e o respeito, também se mostraram benéficas aos relacionamentos, assim como no estudo de Rönnerhag et al. (2019). Porém, por algumas vezes, a confiança dificultou a comunicação entre a equipe, na medida em que se sobressaiu ao cuidado e à atenção necessária para execução de tarefas críticas em razão da confiança em um colega de trabalho. Na teoria da ação comunicativa de Jürgen Habermas (1997), talvez se encontre justificativas para este viés, quando ele traz que ao reconhecer algo como válido, ou acreditar em alguém, tem-se certezas, mas certezas que podem ser muito distintas, além disso acreditar em uma pessoa significa excluir que esta pessoa possa estar pensando algo distinto do que diz.

Outros fatores capazes de afetar a comunicação interpessoal são identificados na literatura, tais quais o conhecimento e experiência (Olde Bekkink et al., 2018). No ambiente cirúrgico, os componentes fundamentais à comunicação eficaz identificados foram: familiaridade da equipe, expectativas de função, comunicação focada em procedimentos, humor do cirurgião, hierarquia percebida e estrutura social e comunicação formal (Grade et al., 2019), todos estes elementos apareceram entre as descobertas desta pesquisa.

Uma revisão integrativa examinou a, igualmente identificados em nosso estudo, encontram-se a comunicação por meio de ferramentas, incluindo diretrizes, protocolos e registros de comunicação e a participação de farmacêuticos em equipes interdisciplinares (Manias, 2018). A participação do farmacêutico apoiando a tomada de decisão do enfermeiro foi evidenciada apenas no $\mathrm{H} @$, cuja integração junto à equipe mostrou-se fundamental.

Desta forma, os dados revelados nesta pesquisa, demonstram a maneira como os enfermeiros têm se relacionado com a equipe de trabalho, podendo auxiliar na compreensão de comportamentos que impactam em eventos adversos, e assim compartilhar formas que encorajem os enfermeiros a manifestarem suas preocupações com a segurança dos pacientes e por conseguinte, mitigar erros. Contudo, mesmo que os achados não possam ser reproduzidos para os diversos ambientes de saúde, dada sua diversidade, complexidade, níveis de atendimento e composição por equipes multidisciplinares, contribuem para o direcionamento de pesquisas futuras, pois, comunicar-se não se trata apenas de um processo individual de tomada de decisão, mas um processo social dinâmico (Raemer et al., 2016).

As limitações do estudo se concentraram no viés de memória dos participantes da pesquisa; nas entrevistas virtuais e gravadas, em virtude da pandemia por COVID-19, impedindo a proximidade entre entrevistador e entrevistado, dificultando a detecção da comunicação não verbal durante as entrevistas, pela interferência do ambiente e do recurso tecnológico utilizado; além, da carga emocional dos profissionais, podendo ter influenciado de forma negativa nas respostas. E ainda, o aspecto de interpretação dos dados tratando-se de um processo indutivo por pesquisadora única.

\section{Conclusão}

Esta pesquisa mostrou a diversidade de fatores favoráveis à capacidade de fala dos enfermeiros frente a situações de risco em curso, praticadas por outros profissionais da saúde, entre os mais citados estiveram os facilitadores pessoal, conhecimento, experiência, liderança, gestão e processos.

Embora numa frequência baixa, mas significativa no que diz respeito à análise de relação, a interdisciplinaridade pareceu positiva sob o ponto de vista de compartilhamento de responsabilidade na tomada de decisão e frente à falta de conhecimento específico, especialmente reconhecida no $\mathrm{H} \odot$, na relação enfermagem-farmacêutico, cuja frequência baixa de 
ocorrência enquanto facilitadora, pode estar vinculada às deficiências de formação dos profissionais da saúde com enfoque interdisciplinar.

Os fatores interferentes na tomada de decisão dos enfermeiros frente aos atos inseguros, ainda são pouco evidenciados na literatura nacional, trazendo à luz a necessidade de estudos futuros. Pois, somado a complexidade do tema comunicação e eventos adversos, que nos permite vários tipos de abordagens, outras variáveis peculiares do sistema de saúde, da cultura e da própria formatação da enfermagem em diferentes níveis de ensino, tornam a temática ainda mais desafiadora em nosso país.

Para tanto, o ponto forte deste estudo, está na compreensão de como as relações interprofissionais afetam o comportamento dos enfermeiros, e por sua vez, interferem na comunicação capaz de promover a segurança do paciente. Os determinantes à decisão de manifestar-se ou não, em prol do paciente, estiveram relacionados à gravidade do dado, ao profissional e ou à pessoa a ser abordada, às características pessoais do manifestante, e a situação ou contexto.

\section{Referências}

Agency for Healthcare Research and Quality. (2017). Estimando os custos adicionais de internação hospitalar e mortalidade associados às condições selecionadas do hospital. AHRQ. https://www.ahrq.gov/professionals/quality-patient-safety/pfp/haccost2017.html.

AHRQ. Agency for Healthcare Research and Quality. (2004). Pesquisa hospitalar sobre cultura de segurança do paciente. Pesquisa Hospitalar Sobre Cultura de Segurança Do Paciente. Rockville: AHRQ. Https://Www.Ahrq.Gov/Sops/Surveys/Hospital/Index.Html.

Bardin, L. (2016). Análise de conteúdo (70th ed.).

Brasil Ministério da saúde. (2013). portaria n 529, de01 de abril de 2013, institui o Programa Nacional de segurança do paciente. Brasília (DF): Ministério Da Saúde.

Campbell, A., Sutcliffe, K., Carlstrom, M., Clements, J., Martin, G. P., Dixon-Woods, M., Aveling, E. L., Willars, J., Tarrant, C., Pronovost, P. J., Gillespie, B., Kang, E., Chaboyer, W., Harbeck, E., Steel, C., Fairweather, N., Schwappach, D., Richard, A., Rönnerhag, M., ... Outcomes, P. (2019). Speaking up behaviors and safety climate in an Austrian university hospital. BMJ Quality and Safety, 38(1), 1-7. https://doi.org/10.1093/intqhc/mzy089

Cunningham, U., Ward, M. E., De Brún, A., \& McAuliffe, E. (2018). Team interventions in acute hospital contexts: A systematic search of the literature using realist synthesis. BMC Health Services Research, 18(1), 1-15. https://doi.org/10.1186/s12913-018-3331-3

Figueiredo, T. W. B., Da Silva, L. A. A., Brusamarello, T., De Oliveira, E. S., Dos Santos, T., \& Pontes, L. (2018). Tipos, Causas E Estratégias De Intervenção Frente a Erros De Medicação: Uma Revisão Integrativa. Revista de Enfermagem e Atenção à Saúde, 7(2), 155-175. https://doi.org/10.18554/reas.v7i2.2494

Grade, M. M., Tamboli, M. K., Bereknyei Merrell, S., Mueller, C., \& Girod, S. (2019). Attending Surgeons Differ from Other Team Members in Their Perceptions of OR Communication. Journal of Surgical Research, 235, 105-112. https://doi.org/10.1016/j.jss.2018.09.030

Hammoudi, B. M., Ismaile, S., \& Abu Yahya, O. (2018). Factors associated with medication administration errors and why nurses fail to report them. Scandinavian Journal of Caring Sciences, 32(3), 1038-1046. https://doi.org/10.1111/scs.12546

Habermas, J. (1997). Teoría de la acción comunicativa: complementos y estudios previos (Cátedra S.A (ed.); $3^{\circ}$ ).

Kiesewetter, I., Könings, K. D., Kager, M., \& Kiesewetter, J. (2018). Undergraduate medical students' behavioural intentions towards medical errors and how to handle them: A qualitative vignette study. BMJ Open, 8(3). https://doi.org/10.1136/bmjopen-2017-019500

Kong, L. N., Zhu, W. F., He, S., Chen, S. Z., Yang, L., Qi, L., \& Peng, X. (2019). Attitudes towards patient safety culture among postgraduate nursing students in China: A cross-sectional study. Nurse Education in Practice, 38(1), 1-6. https://doi.org/10.1016/j.nepr.2019.05.014

Kowalski, Sonya L. MSN, RN, ACNS-BC; Anthony, Maureen PhD, RN CE, AJN. (n.d.). No Title. American Journal of Nursing, Volume 117(Edição 2), p 3448. https://doi.org/10.1097 / 01.NAJ.0000512274.79629.3c

Lee, C. T. S., \& Doran, D. M. (2017). The Role of Interpersonal Relations in Healthcare Team Communication and Patient Safety: A Proposed Model of Interpersonal Process in Teamwork. The Canadian Journal of Nursing Research = Revue Canadienne de Recherche En Sciences Infirmieres, 49(2), 75-93. https://doi.org/10.1177/0844562117699349

Levine, K. J., Carmody, M., \& Silk, K. J. (2020). The influence of organizational culture, climate and commitment on speaking up about medical errors. Journal of Nursing Management, 28(1), 130-138. https://doi.org/10.1111/jonm.12906

Manias, E. (2018). Effects of interdisciplinary collaboration in hospitals on medication errors: an integrative review. Expert Opinion on Drug Safety, 17(3), 259275. https://doi.org/10.1080/14740338.2018.1424830

Martin, G. P., Aveling, E. L., Campbell, A., Tarrant, C., Pronovost, P. J., Mitchell, I., Dankers, C., Bates, D., \& Dixon-Woods, M. (2018). Making soft intelligence hard: A multi-site qualitative study of challenges relating to voice about safety concerns. BMJ Quality and Safety, $27(9)$, 710-717. https://doi.org/10.1136/bmjqs-2017-007579 
Morrow, K. J., Gustavson, A. M., \& Jones, J. (2016). Speaking up behaviours (safety voices) of healthcare workers: A metasynthesis of qualitative research studies. International Journal of Nursing Studies, 64, 42-51. https://doi.org/10.1016/j.ijnurstu.2016.09.014

Olde Bekkink, M., Farrell, S. E., \& Takayesu, J. K. (2018). Interprofessional communication in the emergency department: residents' perceptions and implications for medical education. International Journal of Medical Education, 9, 262-270. https://doi.org/10.5116/ijme.5bb5.c111

Pattni, N., Arzola, C., Malavade, A., Varmani, S., Krimus, L., \& Friedman, Z. (2019). Challenging authority and speaking up in the operating room environment: a narrative synthesis. British Journal of Anaesthesia, 122(2), 233-244. https://doi.org/10.1016/j.bja.2018.10.056

Raemer, D. B., Kolbe, M., Minehart, R. D., Rudolph, J. W., \& Pian-Smith, M. C. M. (2016). Improving anesthesiologists' ability to speak up in the operating room: A randomized controlled experiment of a simulation-based intervention and a qualitative analysis of hurdles and enablers. Academic Medicine, 91(4), 530-539. https://doi.org/10.1097/ACM.0000000000001033

Rönnerhag, M., Severinsson, E., Haruna, M., \& Berggren, I. (2019). A qualitative evaluation of healthcare professionals’ perceptions of adverse events focusing on communication and teamwork in maternity care. Journal of Advanced Nursing, 75(3), 585-593. https://doi.org/10.1111/jan.13864

Schwappach, D., Sendlhofer, G., Kamolz, L. P., Köle, W., \& Brunner, G. (2019). Speaking up culture of medical students within an academic teaching hospital: Need of faculty working in patient safety. PLoS ONE, 14(9), 1-13. https://doi.org/10.1371/journal.pone.0222461

TartagliaReis, C., Paiva, S., \& Sousa, P. (2018). The patient safety culture: A systematic review by characteristics of Hospital survey on patient safety culture dimensions. International Journal for Quality in Health Care, 30(9). https://doi.org/10.1093/intqhc/mzy080

Varallo, F. R., Passos, A. C., Nadai, T. R. de, \& Mastroianni, P. de C. (2018). Incidents reporting: barriers and strategies to promote safety culture. Revista Da Escola de Enfermagem Da U S P, 52, e03346. https://doi.org/10.1590/S1980-220X2017026403346

Yin, R.K. (2015). O estudo de caso. Porto Alegre: Bookman. 